\title{
The BAIKAL neutrino experiment-Physics results and perspectives
}

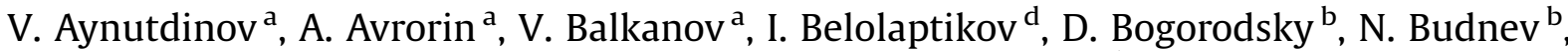

I. Danilchenko ${ }^{a}$, G. Domogatsky ${ }^{a}$, A. Doroshenko ${ }^{a}$, A. Dyachok ${ }^{b}$, Zh.-A. Dzhilkibaev ${ }^{a}$, S. Fialkovsky $^{\mathrm{f}}$, O. Gaponenko ${ }^{\text {a }}$, K. Golubkov ${ }^{\text {d }}$, O. Gress ${ }^{\text {b }}$, T. Gress ${ }^{\text {b }}$, O. Grishin ${ }^{\text {b }}$, A. Klabukov ${ }^{\text {a }}$, A. Klimov ${ }^{\text {, }}$, A. Kochanov ${ }^{\text {b }}$, K. Konischev ${ }^{\text {d }}$, A. Koshechkin ${ }^{a}$, V. Kulepov ${ }^{f}$, D. Kuleshov ${ }^{\text {a }}$, L. Kuzmichev ${ }^{c}$, S. Lovtsov $^{\text {b }}$, E. Middell ${ }^{\text {e }}$, S. Mikheyev ${ }^{\text {a }}$, M. Milenin ${ }^{f}$, R. Mirgazov ${ }^{\text {b }}$, E. Osipova ${ }^{c}$, G. Pan'kov $^{\mathrm{b}}$, L. Pan'kov $^{\mathrm{b}}$, A. Panfilov $^{\mathrm{a}}$, D. Petukhov ${ }^{a}$, E. Pliskovsky ${ }^{d}$, P. Pokhil ${ }^{a}$, V. Poleschuk ${ }^{a}$, E. Popova ${ }^{c}$, A. Rastegin $^{\mathrm{b}}$, V. Prosin ${ }^{\mathrm{c}}$, M. Rozanov ${ }^{g}$, V. Rubtzov ${ }^{b}$, A. Sheifler ${ }^{a}$, A. Shirokov ${ }^{c}$, B. Shoibonov ${ }^{\text {d }}$, Ch. Spiering ${ }^{\text {e }}$ O. Suvorova ${ }^{\mathrm{a}}$, B. Tarashansky ${ }^{\text {b }}$, R. Wischnewski ${ }^{\text {e,* }}$, I. Yashin ${ }^{\text {c }}$, V. Zhukov ${ }^{\text {a }}$

a Institute for Nuclear Research, 60th October Anniversary pr. 7a, Moscow 117312, Russia

${ }^{\mathrm{b}}$ Irkutsk State University, Irkutsk, Russia

' Skobeltsyn Institute of Nuclear Physics MSU, Moscow, Russia

d Joint Institute for Nuclear Research, Dubna, Russia

e DESY, Zeuthen, Germany

${ }^{f}$ Nizhni Novgorod State Technical University, Nizhni Novgorod, Russia

${ }^{g}$ St. Petersburg State Marine University, St. Petersburg, Russia

${ }^{\mathrm{h}}$ Kurchatov Institute, Moscow, Russia

\section{A R T I C L E I N F O}

\section{Available online 16 December 2008}

Keywords:

Neutrino telescopes

Neutrino astronomy

UHE neutrinos

BAIKAL

\begin{abstract}
A B S T R A C T
We review the status of the Lake Baikal Neutrino Experiment. The Neutrino Telescope NT200 has been operating since 1998 and has been upgraded to the 10 Mton detector NT200+ in 2005. We present selected astroparticle physics results from long-term operation of NT200. Also discussed are activities towards acoustic detection of UHE-energy neutrinos, and results of associated science activities. Preparation towards a km3-scale (Gigaton volume) detector in Lake Baikal is currently a central activity. As an important milestone, a km3-prototype string, based on completely new technology, has been installed and is operating together with NT200+ since April, 2008.
\end{abstract}

( 2008 Elsevier B.V. All rights reserved.

\section{Introduction}

The Baikal Neutrino Telescope NT200 has been taking data since 1998; its first stage telescope NT36 was-back in 1993-the first underwater Cherenkov neutrino detector. Since 2005, the upgraded 10-Mton scale detector NT200+ is in operation. Detector configuration and performance have been described elsewhere [1-6]. The most recent milestone of the ongoing $\mathrm{km} 3-$ telescope research and development work (R\&D) was the installation of a "new technology" prototype string in spring 2008, operating now as part of NT200+. Fig. 1 gives a sketch of the current status of the telescope NT200+, including the km3prototype string.

In this paper we review astroparticle physics results obtained with NT200, a feasibility study on acoustic neutrino detection, and the R\&D activities towards a km3-scale Baikal telescope.

\footnotetext{
*Corresponding author. Tel.: +493376277348; fax: +493376277330

E-mail address: wischnew@ifh.de (R. Wischnewski).
}

The success of the Baikal neutrino experiment is, to a considerable degree, related to the favorable natural conditions of the site-thus site studies and related science were always an integral part of the project. We will discuss some of the relevant problems for the lake's ecosystem, which were studied through the use of new technologies, instruments and methods designed in the framework of the Baikal experiment. We will also mention future options.

\section{Selected physics results from NT200}

\subsection{Atmospheric neutrinos}

The signature of charged current muon neutrino events is a muon crossing the detector from below. Muon track reconstruction algorithms and background rejection have been described elsewhere [8]. Compared to Ref. [8], the analysis of the five-year sample (April 1998-February 2003, 1008 days live time) was 
optimized for higher signal passing rate, i.e. accepting a slightly higher contamination of $\sim 20 \%$ fake events [9]. A total of 372 upward going neutrino candidates were selected. From MonteCarlo simulation a total of 385 atmospheric neutrino and background events are expected, with a median muon-angular resolution of $2.2^{\circ}$. The skyplot for this event sample is shown in Fig. 2.

For the $v_{\mu}$-analysis procedure, a standard three-dimensional muon reconstruction is performed for all events [8]. The good agreement of the obtained downward muon-angular distribution with $\mathrm{MC}$ is shown in Fig. 3 (right) (see also earlier Baikal flux measurements [1]). All events reconstructed as upgoing are treated in a second pass as neutrino candidates, to additionally filter out fake events. The zenith-angular distribution of upward reconstructed neutrino events is given in Fig. 3 (left), for two different choices of the background content $(S / N \sim 3$ and $\sim 10$, respectively), as used for different analyses. In Fig. 3 also shown are MC predictions for the sum of $v$-signal and background

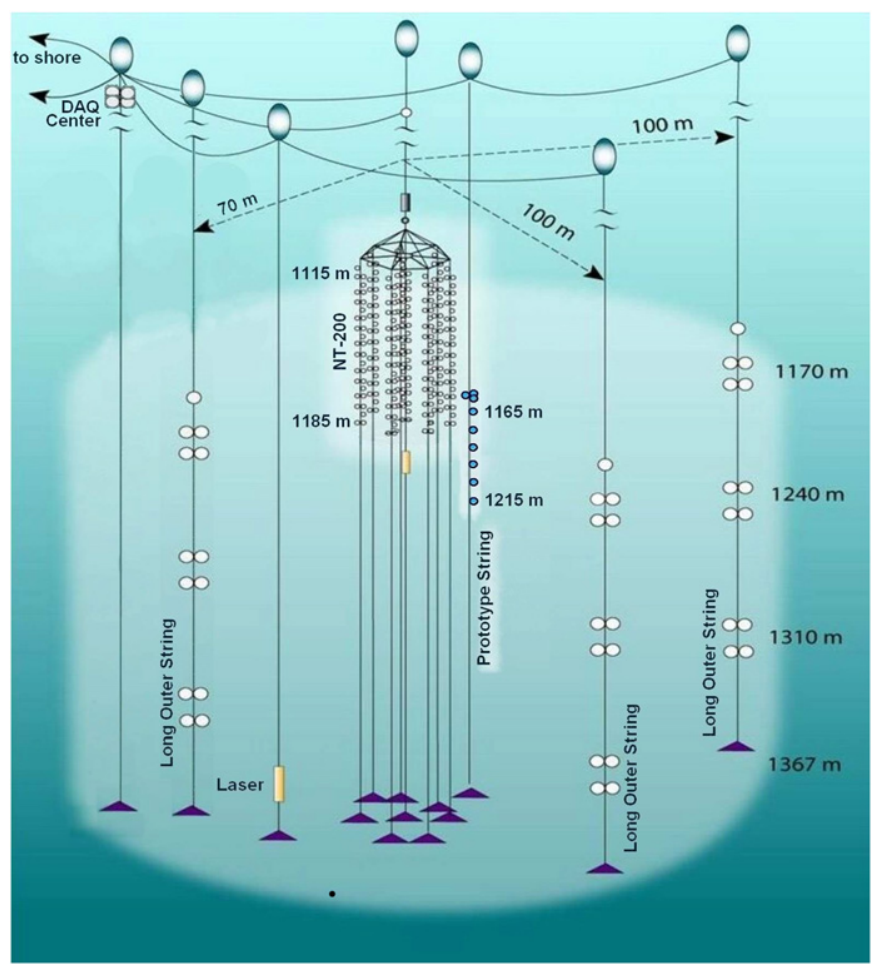

Fig. 1. The Baikal Telescope NT200+ as of 2008: the compact NT200 (center), three long outer strings and the new technology km3-prototype string.

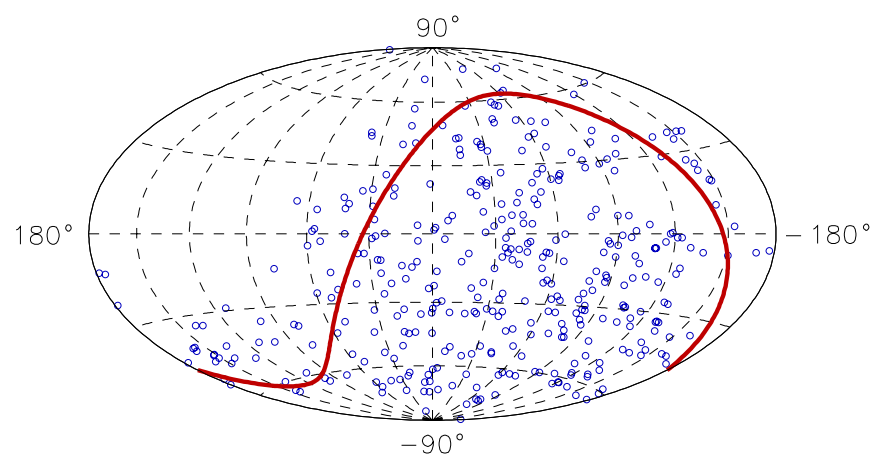

Fig. 2. Skyplot (galactic coordinates) of neutrino events for five years. The solid curve shows the equator. without and with $v$-oscillations (see Section 2.2 for parameters), as well as for the background (histograms from top to bottom, respectively). Data and MC are in good agreement, given the known systematic uncertainties of absolute atmospheric $v$-flux predictions.

\subsection{Search for neutrinos from WIMP annihilation}

The search for WIMPs annihilating in the Earth center with the Baikal neutrino telescope is based on a possible signal of nearly vertically upward going muons, exceeding the flux of atmospheric neutrinos. Signal event selection relies on a series of cuts which are tailored to the response of the telescope to nearly vertically upward moving muons [10]. These cuts select muons with $-1<\cos (\theta)<-0.75$ and result in a detection area of about $1800 \mathrm{~m}^{2}$ for vertically upward going muons.

The energy threshold for this analysis is $E_{\mathrm{thr}} \sim 10 \mathrm{GeV}$ i.e. lower than for the standard $v_{\mu}$-analysis described in Section 2.1 $\left(E_{\mathrm{thr}} \sim 15-20 \mathrm{GeV}\right)$.

From 1038 live time days (1998-2003), 48 events with $-1<\cos (\theta)<-0.75$ have been selected as clear neutrino events, compared to 56.6 events expected from an atmospheric neutrino MC (Bartol-96 flux [11] with oscillation parameters $\delta m^{2}=2.5 \times$ $10^{-3} \mathrm{eV}^{2}$ and full mixing, $\theta_{m} \approx \pi / 4$; without oscillation 73.1 MC-events are expected); for details see Refs. [12,13]. We find that absolute number and zenith-angular distributions for MC and experimental data are within statistical uncertainties in good agreement.

Regarding the 48 detected events as being induced by atmospheric neutrinos, one can derive an upper limit on the additional flux of muons from the center of the Earth due to annihilation of neutralinos - the favored candidate for cold dark matter. From this, we obtain $90 \%$ CL muon flux limits for six cones around the opposite zenith $\left(E_{\mathrm{thr}}>10 \mathrm{GeV}\right)$. From the strong dependence of the size of the cone on the neutralino mass, see Refs. [14-16], we calculate $90 \%$ CL flux limits as function of neutralino mass. A correction is applied for each neutralino mass to translate from the experimental 10 to $1 \mathrm{GeV}$ threshold. These limits are shown in Fig. 4. Also shown are limits obtained by Baksan [14], MACRO [15], Super-Kamiokande [16] and AMANDA (from the hard neutralino annihilation channels) [17].

\subsection{A search for fast magnetic monopoles}

Fast magnetic monopoles with Dirac charge $g=68.5 e$ are interesting objects to search for with deep underwater neutrino telescopes. The intensity of monopole Cherenkov radiation (for $\beta=1$ ) is $\approx 8300$ times higher than that of muons. Optical modules (OMs) of the Baikal experiment can detect such an object from a distance of up to hundred meters. The processing chain for fast monopoles starts with the selection of events with a high multiplicity of hit channels: $N_{\text {hit }}>30$. Because of the high background of downward atmospheric muons, we restrict the search to upward moving monopoles. For an upward going particle, the time tags of hit channels increase with rising $z$-coordinates from bottom to top of the detector. To suppress downward moving particles, a cut on the value of the time$z$-correlation, $C_{t z}$, is applied.

Within 1003 days of live time used in this analysis, about $3.3 \times$ $10^{8}$ events with $N_{\text {hit }} \geqslant 4$ have been recorded, with 20943 of them satisfying cut $0\left(N_{\text {hit }}>30\right.$ and $\left.C_{t z}>0\right)$. For further background suppression (see Ref. [18] for details of the analysis) we use additional cuts, which essentially reject muon events and at the same time only slightly reduce the effective area for relativistic monopoles. 

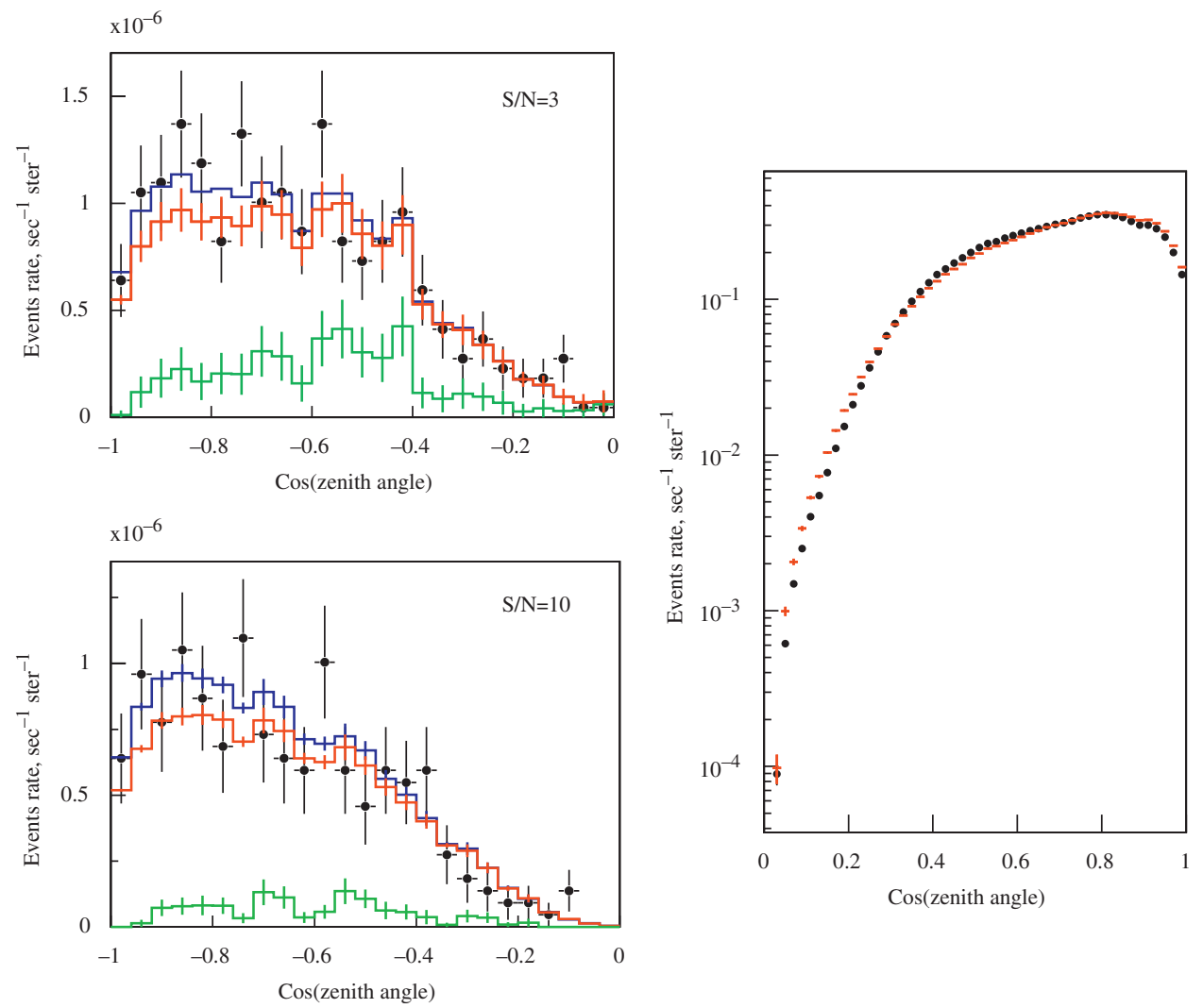

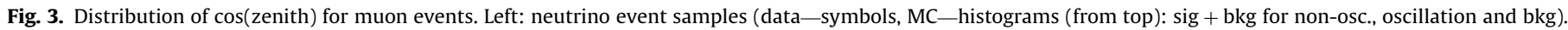
Right: downward atmospheric muons (data—symbols, MC—histogram).

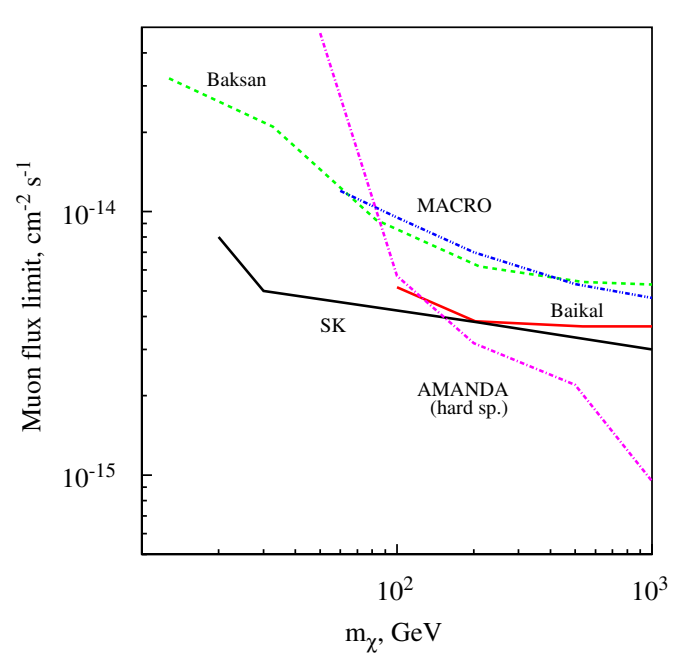

Fig. 4. Limits on the excess of muon flux from the center of the Earth as a function of neutralino mass.

The upper limit on a flux of magnetic monopoles with $\beta=1$ is $4.6 \times 10^{-17} \mathrm{~cm}^{-2} \mathrm{~s}^{-1} \mathrm{sr}^{-1}$. In Fig. 5 we compare our upper limit for an isotropic flux of fast monopoles obtained with the Baikal neutrino telescope to the limits from the underground experiments Ohya [19] and MACRO [20], and from the underice detectors AMANDA-B10 [21] and AMANDA-II [22] (preliminary).

We mention, that with an early NT200 prototype, a search for slow monopoles has been published [1] $\left(\beta=10^{-5}-10^{-3}\right.$ and for monopole catalyzed proton decay). An NT200-analysis, with improved sensitivity down to $10^{-17} \mathrm{~cm}^{-2} \mathrm{~s}^{-1} \mathrm{sr}^{-1}$, is in preparation.

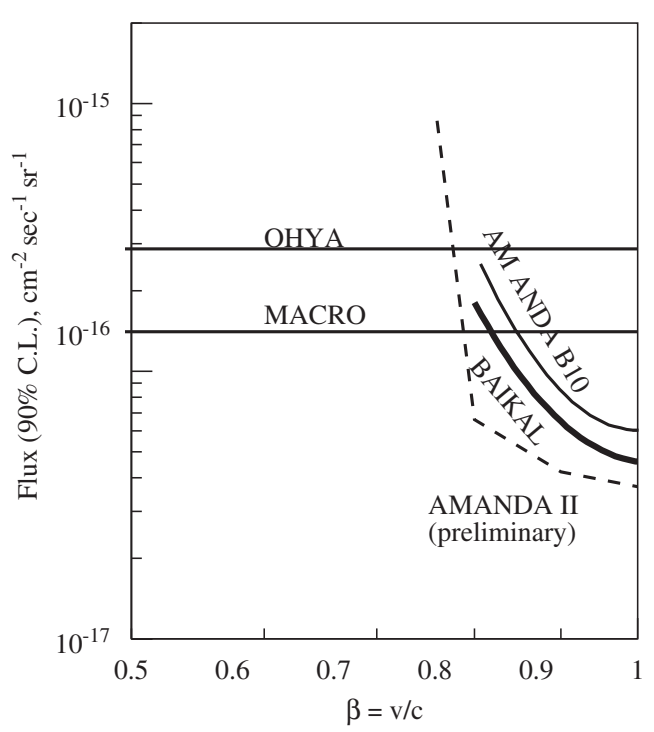

Fig. 5. Upper limits on the flux of fast monopoles obtained in this analysis (Baikal) and in other experiments.

\subsection{A search for extraterrestrial high-energy neutrinos}

The BAIKAL survey for high-energy neutrinos searches for bright cascades produced at the neutrino interaction vertex in a large volume around the neutrino telescope [3]. We select events with high multiplicity of hit channels $N_{\text {hit }}$, corresponding to bright cascades. To separate high-energy neutrino events from background events, a cut to select events with upward moving light signals has been developed. We define for each event 
$t_{\min }=\min \left(t_{i}-t_{j}\right)$, where $t_{i}, t_{j}$ are the arrival times at channels $i, j$ on each string, and the minimum over all strings is calculated. Positive and negative values of $t_{\min }$ correspond to upward and downward propagation of light, respectively.

Within the 1038 days of the detector live time, $3.45 \times 10^{8}$ events with $N_{\text {hit }} \geqslant 4$ have been recorded. For this analysis we used 22597 events with hit channel multiplicity $N_{\text {hit }}>15$ and $t_{\min }>-10 \mathrm{~ns}$. We conclude that data are consistent with simulated background for both $t_{\min }$ and $N_{\text {hit }}$ distributions. No statistically significant excess above the background from atmospheric muons has been observed. To maximize the sensitivity to a neutrino signal we introduce a cut in the $\left(t_{\min }, N_{\text {hit }}\right)$ phase space.

Since no events have been observed which pass the final cuts, upper limits on the diffuse flux of extraterrestrial neutrinos are calculated. For a 90\% confidence level an upper limit on the number of signal events of $n_{90 \%}=2.5$ is obtained assuming an uncertainty in signal detection of $24 \%$ and a background of zero events.

A model of astrophysical neutrino sources, for which the total number of expected events, $N_{m}$, is larger than $n_{90 \%}$, is ruled out at 90\% CL. Table 1 represents event rates and model rejection factors (MRF) $n_{90 \%} / N_{m}$ for models of astrophysical neutrino sources obtained from our search, as well as MRF obtained recently by the AMANDA collaboration [23-25].

For an $E^{-2}$ behavior of the neutrino spectrum and a flavor ratio $v_{e}: v_{\mu}: v_{\tau}=1: 1: 1$, the $90 \% \mathrm{CL}$ upper limit on the neutrino flux of all flavors obtained with the Baikal neutrino telescope NT200 is

$E^{2} \Phi<8.1 \times 10^{-7} \mathrm{~cm}^{-2} \mathrm{~s}^{-1} \mathrm{sr}^{-1} \mathrm{GeV}$

for $20 \mathrm{TeV}<E_{v}<50 \mathrm{PeV}$. For the resonant process at the resonance neutrino energy $E_{0}=6.3 \times 10^{6} \mathrm{GeV}$ the model-independent limit on $\bar{v}_{e}$ is

$\Phi_{\bar{v}_{e}}<3.3 \times 10^{-20} \mathrm{~cm}^{-2} \mathrm{~s}^{-1} \mathrm{sr}^{-1} \mathrm{GeV}^{-1}$.

Fig. 6 shows our upper limit on the all flavor $E^{-2}$ diffuse flux (Eq. (1)) as well as the model-independent limit on the resonant $\bar{v}_{e}$ flux ("*”) (Eq. (2)). Also shown are the limits obtained by AMANDA [23-25] and MACRO [34], theoretical bounds obtained by Berezinsky (model independent (B) [35] and for an $E^{-2}$ shape of the neutrino spectrum $\left(\mathrm{B}\left(E^{-2}\right)\right)[36]$, by Waxman and Bahcall (WB) [37], by Mannheim et al. (MPR) [31], predictions for neutrino fluxes from topological defects (TD) [32], prediction on diffuse flux from AGNs according to Nellen et al. (NMB) [38], as well as the atmospheric conventional neutrino fluxes [39] from horizontal and vertical directions $((v)$ upper and lower curves, respectively) and atmospheric prompt neutrino fluxes $\left(v_{p r}\right)$ from Volkova et al. [40].

Table 1

Expected number of events $N_{m}$ and experimental model rejection factors for astrophysical neutrino source models.

\begin{tabular}{lclll}
\hline Model & \multicolumn{2}{c}{ BAIKAL [3] } & & AMANDA [23-25] \\
\cline { 2 - 3 } \cline { 5 - 5 } & $v_{e}+v_{\mu}+v_{\tau}$ & $n_{90 \%} / N_{m}$ & & $n_{90 \%} / N_{m}$ \\
\hline $0^{-6} \times E^{-2}$ & 3.08 & 0.81 & 0.22 \\
SS Quasar [26] & 10.00 & 0.25 & 0.21 \\
SS05 Quasar [27] & 1.00 & 2.5 & 1.6 \\
SP u [28] & 40.18 & 0.062 & 0.054 \\
SP 1 [28] & 6.75 & 0.37 & 0.28 \\
P p $\gamma$ [29] & 2.19 & 1.14 & 1.99 \\
M pp + p $\gamma$ [30] & 0.86 & 2.86 & 1.19 \\
MPR [31] & 0.63 & 4.0 & 2.0 \\
SeSi [32] & 1.18 & 2.12 & - \\
\hline
\end{tabular}

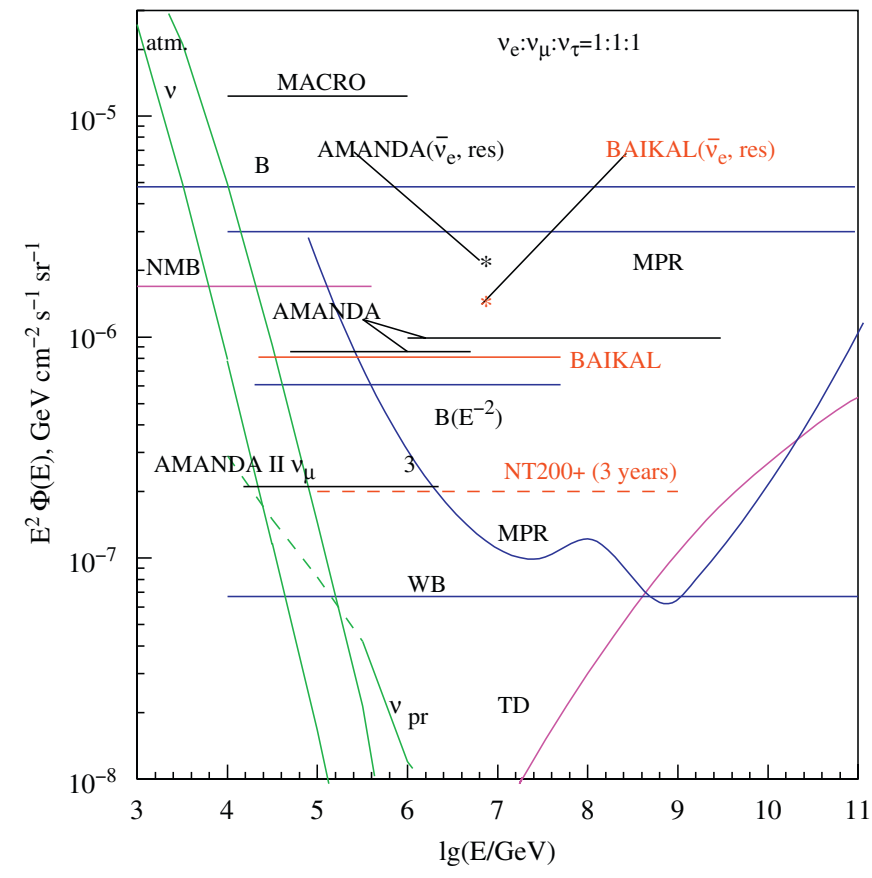

Fig. 6. All flavor neutrino flux predictions in different models of neutrino sources compared to experimental upper limits to $E^{-2}$ fluxes obtained by this analysis and other experiments (see text). Also shown is the sensitivity expected for three live years of the new telescope NT200+ $[5,33]$.

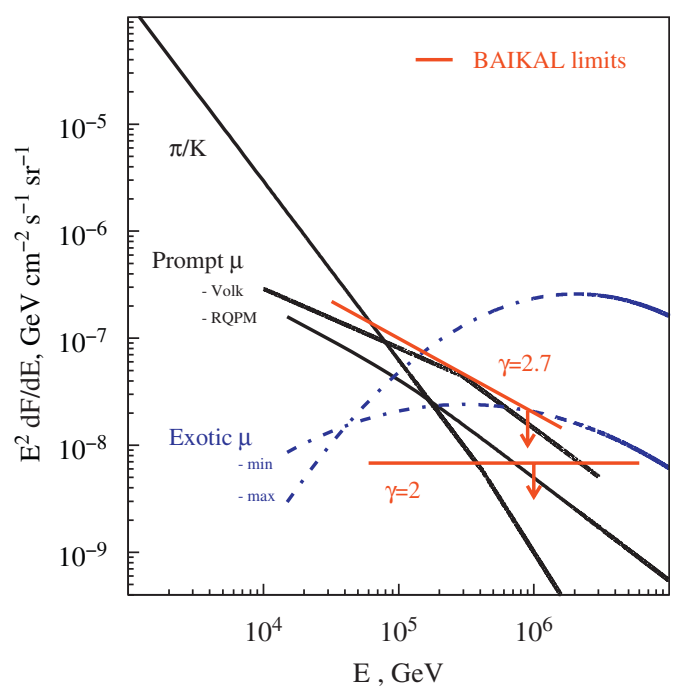

Fig. 7. Obtained upper limits on the high-energy atmospheric muon flux (curves with arrows for $\gamma=2 ; 2.7$ ); and predicted fluxes (for $\pi / \mathrm{K}$, prompt; and for exotic).

\subsection{A search for high-energy muons}

To search for high-energy muons, we use the same event sample and cuts as for the diffuse high-energy neutrino search (see above; analysis restricted to 508 live days), to which atmospheric muons in turn are a background. With no events left at final cut level, upper flux limits can be derived for various muon energy spectra. Fig. 7 shows the experimental limit for a typical prompt muon spectrum with $\gamma=2.7$, together with theoretical predictions $[2,40,41]$.

We can also test for an "exotic" component of high-energy atmospheric muons, which had been postulated to explain the "knee" in the cosmic ray energy spectrum by a new interaction at PeV-scale (see Refs. $[9,42,43]$ and ref. therein). For such hard 
exotic spectra (dashed curves in Fig. 7), NT200 has a high sensitivity. Our experimental limit [42] for a generic $E^{-2}$-spectrum is given in Fig. 7, demonstrating the sensitivity to exclude even the lowest exotic flux.

\section{A prototype device for acoustic neutrino detection in Lake Baikal}

To detect UHE astrophysical neutrinos $\left(\mathrm{E}_{v}>10^{17} \mathrm{eV}\right)$, detectors with sensitive volumes much beyond $\mathrm{km} 3$-scale are needed because of the steeply falling energy spectra. Acoustic detection technology, aiming at registration of the acoustic bipolar signals emitted by UHE-cascades, is a promising technique-and has been investigated since several years in Lake Baikal $[44,45]$. Particularly interesting for our site is the very large acoustic absorption length for $\sim 30 \mathrm{kHz}$ (the peak frequency of acoustic signals), which for freshwater is in the km-range, almost 100 times larger than that of Cherenkov radiation $[45,46]$.

We performed a series of long-term hydro-acoustic measurements to quantify the ambient acoustic noise, which constitutes the main background for acoustic neutrino detection. We found, at stationary and homogeneous meteorological conditions, that the integral noise power in the relevant frequency range $20-50 \mathrm{kHz}$ reaches levels as low as $\sim 1 \mathrm{mPa}[44,47]$, one of the lowest levels measured at currently considered acoustic neutrino detector sites.

Extraction of small signals from background requires an antenna, consisting of a set of hydrophones. We have constructed a digital hydro-acoustic device with four input channels shown in Fig. 8, for stationary common operation with NT200+. It has been installed in April 2006 at one of the telescope moorings at $150 \mathrm{~m}$ depth [45].

Fig. 9 presents an event with bipolar pulses in all four channels, detected by this device. After directional reconstruction, we find that most pulses arrive from the vicinity of the horizontal plane [45]. From the region within $45^{\circ}$ around the opposite zenith, i.e. from the deep lake zone below the device, no events with bipolar pulse form have been observed.

We note that the water temperature at depths below $400 \mathrm{~m}$ is very stable around $\sim 3.4-3.6^{\circ} \mathrm{C}$, see Fig. 10 for a spring 2008 measurement and Section 5 for more details. As also shown in Fig. 10, the temperature is only equal to that of maximal density (TMD—dashed curve [48]) at shallow depths below 200 m; at

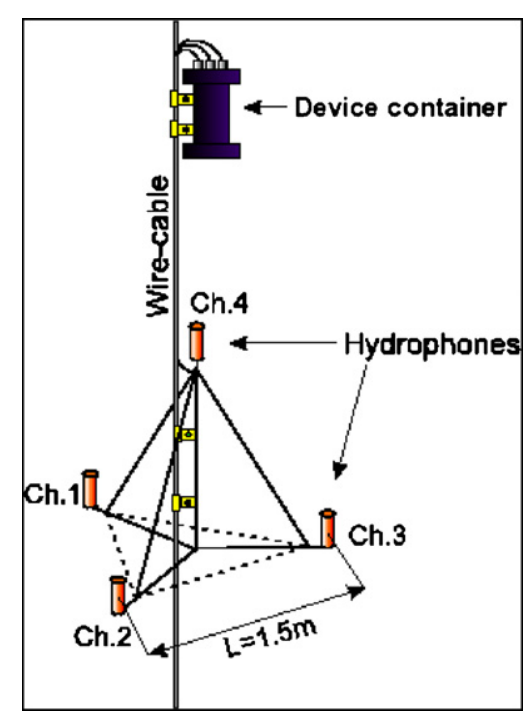

Fig. 8. The stationary four-channel acoustic device, operating in Lake Baikal.

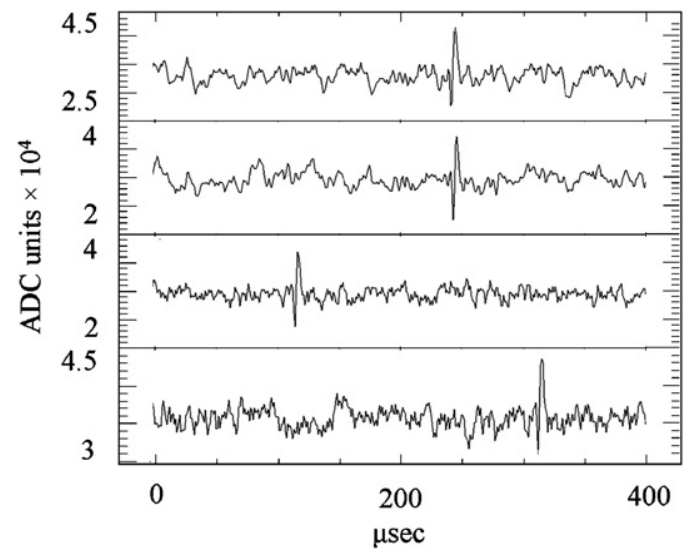

Fig. 9. A triggered event with bipolar pulses (channels 1-4).

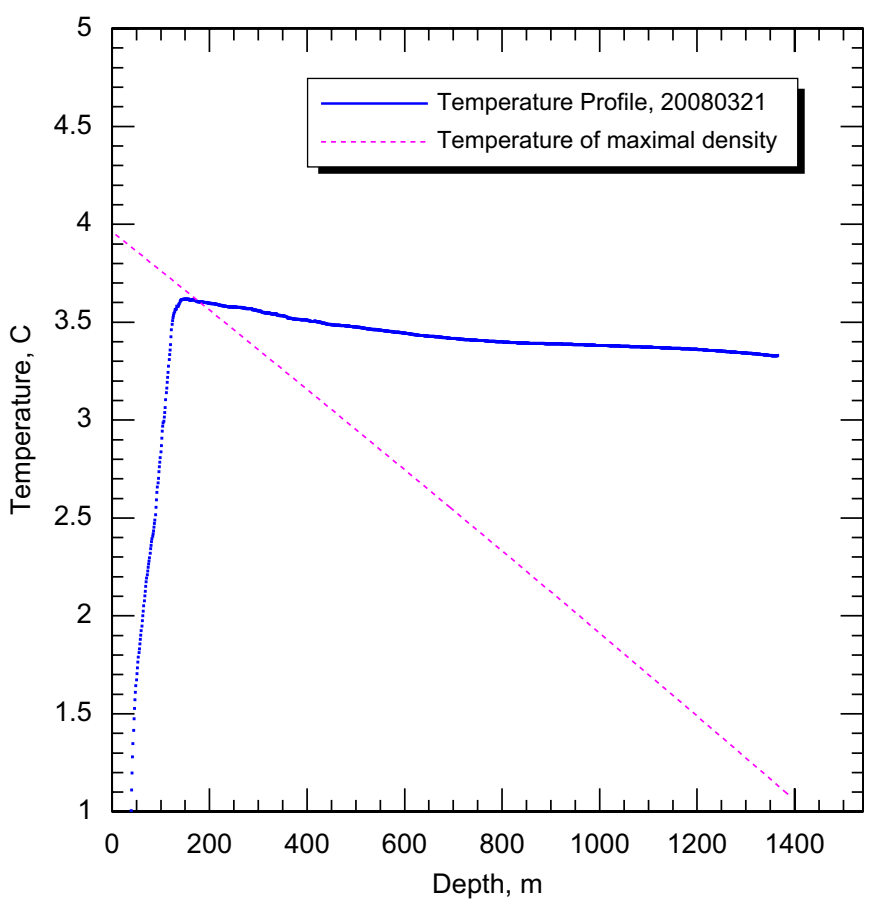

Fig. 10. Measured temperature as function of depth (28.3.2008) and temperature of maximal density, TMD.

larger depths they differ significantly, since TMD falls steeply (by $\sim 0.2{ }^{\circ} \mathrm{C}$ per $100 \mathrm{~m}$ depth).

\section{Towards a km3-detector in Lake Baikal: the new technology string}

The Baikal collaboration has followed over several years an $\mathrm{R} \& \mathrm{D}$ program for an $\mathrm{km} 3$-scale neutrino telescope in Lake Baikal. The construction of NT200+ was a first step in this direction. The existing NT200+ is a natural laboratory to verify many new key elements and design principles of the new telescope.

A Baikal km3-detector could be made of building blocks similar to NT200+, but with NT200 replaced by a single string, still allowing separation of high-energy neutrino induced cascades from background [5]. It will contain a total of 1300-1700 OMs, arranged at 90-100 strings with 12-16 OMs each, and an instrumented length of 300-350 m. Interstring distances will be $\sim 100 \mathrm{~m}$. The effective volume for cascade events above $100 \mathrm{TeV}$ is $0.5-0.8 \mathrm{~km}^{3}$, the threshold for muons is $20-50 \mathrm{TeV}$. 
The most recent $\mathrm{km} 3$-milestone was the construction and installation of a "new technology" prototype string in spring 2008. This string is operating as an integral part of NT200+. Prototype string design and first results are described in detail in these proceedings. It is based on several new technology elements: (1) large area hemispherical PMTs (12 in. Photonis and $13 \mathrm{in.}$ Hamamatsu, first time used in an underwater telescope), (2) $200 \mathrm{MHz}$ FADC readout technology, combined with string-based triggering and time synchronization by array trigger time-stamps, and (3) new calibration elements. Data collection and transmission is based on copper cables using proven DSL-technology (as in NT200+ [5]).

First calibration and verification tests have been successful. The string is now running in either of two operation modes: standalone atmospheric muon trigger, or in coincidence mode with NT200+ (high-energy cascades). A detailed verification of the prototype string will be based on this large statistics data sample.

MC optimization for the $\mathrm{km} 3$-detector design is going on, as well as studies for optimal trigger technologies. A technical design report for the new telescope is due for fall 2008.

\section{Related science with the Baikal telescope}

Related science regarding water parameters, water exchange and deep-water renewal processes [49] are an integral part of the neutrino project. Optical properties (absorption, scattering) are permanently monitored by custom-made devices $[1,50]$ while the background light recorded by the telescope's three-dimensional light sensor array contains rich information on seasonal changes of water luminescense, and even allows to trace short-term flows of luminescent matter [51,52]. The telescope's modern infrastructure offers a natural opportunity to add realtime sensor systems (e.g. seismic, temperature), either connected by cables or underwater acoustic links [53].

The lake's temperature profile is a driving factor for hydrophysical as well as hydrobiological and hydrochemical processes; and at the same time the water temperature is a very efficient tracer to study hydrophysical phenomena in this natural basin. Stationary long-term high-precision temperature measurements have been carried out since March 1999 in cooperation with the Swiss Federal Institute of Environmental Science and Technology (EAWAG). More than 50 temperature loggers, distributed between the lake bottom and $15 \mathrm{~m}$ depth at 3 moorings in the vicinity of NT200+, record the three-dimensional temperature profile all year round at $10 \mathrm{~min}$ intervals with a resolution better than $0.002{ }^{\circ} \mathrm{C}[54,55]$.

The largest seasonal temperature variations occur at shallow depths $<200 \mathrm{~m}$, as discussed in Ref. [55]. Temperatures measured at depths $300-1300 \mathrm{~m}$ in 2005 are, as an example, shown in Fig. 11. The temperature below $300 \mathrm{~m}$ slowly decreases with depth and the density of water is perfectly stratified all around the year. Almost simultaneous temperature variations down to $1000 \mathrm{~m}$ depth are observed. The structures of these variations at different depths clearly correlate; their amplitude decreases with depth [55]. These disturbances have a maximum magnitude close to the autumn homothermy and are connected with intrusion of water from upper layers.

However, regularly observed huge cold-water intrusions into the near-bottom zone (occurring around the time of convective surface mixing in June and December/January), which have a typical volume of about $10 \mathrm{~km}^{3}$, could not be explained by this vertical water exchange, since data did not show vertical disturbances to reach down to the bottom with significant amplitude. These intrusions play a crucial role in oxygenation of

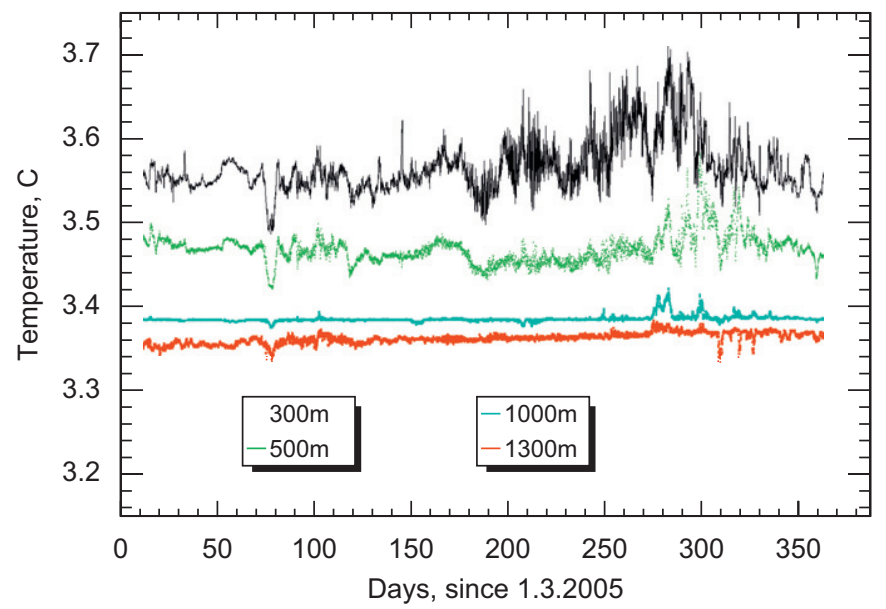

Fig. 11. Vertical temperature profile from $300 \mathrm{~m}$ (top curve) to $1300 \mathrm{~m}$ depth (bottom curve; $\sim 50 \mathrm{~m}$ above lake-bed), as measured during 2005.

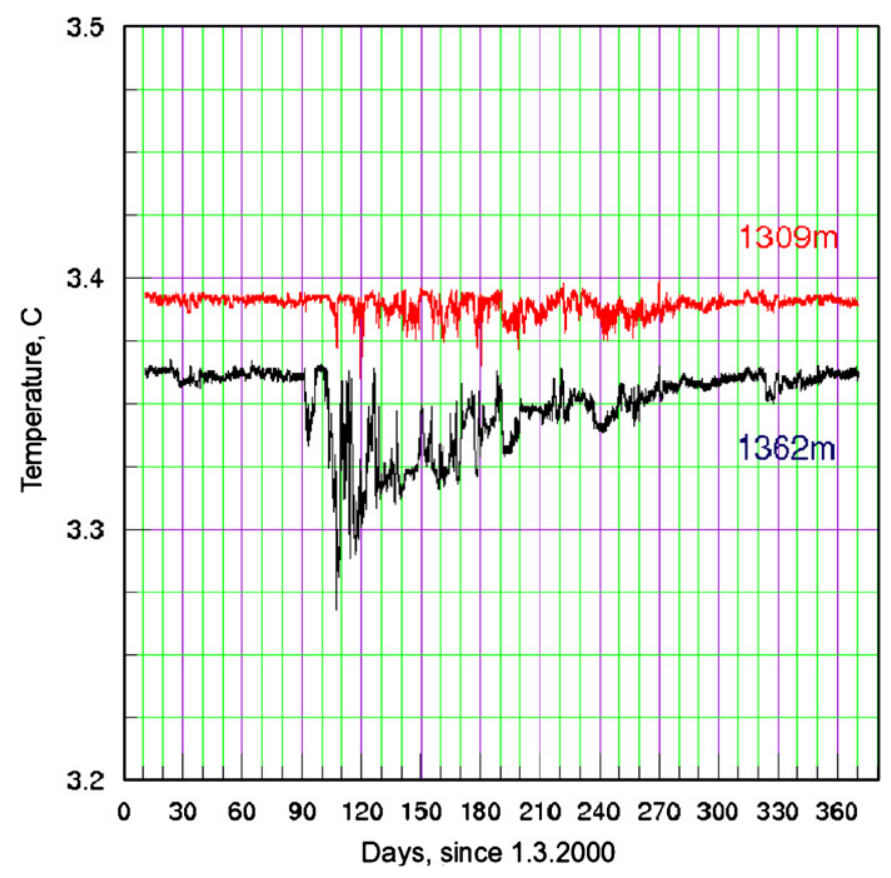

Fig. 12. Temperature at 1309 and $1362 \mathrm{~m}$ depth ( 4 and $57 \mathrm{~m}$ above bottom), measured from March 2000-March 2001. For the huge cold-water intrusion in June, see text

deepest layers and lead to an efficient recycling of the nutrients from the deep water. But up to now, a convincing explanation of this phenomenon had been missing. One of the largest such intrusions was observed in June 2000, when the temperature at $1362 \mathrm{~m}$ depth (just above the bottom) suddenly decreased by almost $0.1^{\circ} \mathrm{C}$ and returned to its initial value only after six months (see Fig. 12). It has been the three-dimensional time-resolved temperature monitoring, that gave the first experimental proof that these advective intrusions are caused by coastal downwelling and subsequent thermobaric instability along the steep lake shores-thus solving a longstanding mystery [54,55].

\section{Conclusion}

The Baikal neutrino telescope NT200 has been taking data since April 1998. The upper limit obtained for a diffuse 
astrophysical $\left(v_{e}+v_{\mu}+v_{\tau}\right) E^{-2}$-flux is $E^{2} \Phi=8.1 \times 10^{-7} \mathrm{~cm}^{-2} \mathrm{~s}^{-1}$ $\mathrm{Sr}^{-1} \mathrm{GeV}$. The limits on the flux of fast magnetic monopoles and on a muon flux induced by WIMP annihilation at the center of the Earth belong to the most stringent limits existing to date. The limit on a $\overline{v_{e}}$ flux at the resonant energy $6.3 \times 10^{6} \mathrm{GeV}$ is presently the most stringent. NT200 also has a high sensitivity for exotic UHE atmospheric muon fluxes.

The significantly upgraded telescope NT200+, a detector with about 5 Mton enclosed volume, has been operating since April 2005, and has an improved sensitivity for a diffuse flux of extraterrestrial neutrinos [5,33]. An ongoing feasibility study for acoustic UHE neutrino detection with a stationary antenna suggests that favorable conditions exist at Lake Baikal.

Multidisciplinary science activities have focused on water monitoring (optical absorption/scattering, luminescense) and high-precision temperature measurements at stationary moorings over almost a decade. These have led to deeper insight into the Lake's ecosystem phenomena. An upgrade to a realtime ecosystem-underwater data network is feasible.

For the planned km3-scale neutrino detector in Lake Baikal, R\&D-activities are in progress. An important km3-milestone was the Spring 2008 installation of a new technology km3-prototype string, with full FADC readout and large area hemisperical PMTs (12 in./13 in.), which now operates together with NT200+. The km3-detector Technical Design Report is planned for fall 2008.

\section{Acknowledgments}

This work was supported by the Russian Ministry of Education and Science, the German Ministry of Education and Research, REC-17 BAIKAL and the Russian Fund of Basic Research (Grants 08-02-00432, 08-02-10010, 07-02-00791, 08-02-00198, 08-0210001), and by the Grant of President of Russia NSh-4580.2006.2. and by NATO-Grant NIG-9811707(2005).

\section{References}

[1] I. Belolaptikov, et al., Astropart. Phys. 7 (1997) 263.

[2] V. Aynutdinov, et al., Nucl. Phys. (Proc. Suppl.) B143 (2005) 335.

[3] V. Aynutdinov, et al., Astropart. Phys. 25 (2006) 140.

[4] V. Aynutdinov, et al., Phys. At. Nucl. 69 (11) (2006) 1914.

[5] V. Aynutdinov, et al., Nucl. Instr. and Meth. A567 (2006) 433.

[6] V. Aynutdinov, et al., in: Proceedings of the 30th ICRC (icrc1084), Merida, Mexico2007 arXiv.org: astro-ph/0710.3063.

[8] V. Balkanov, et al., Astropart. Phys. 12 (1999) 75.

[9] V. Aynutdinov, et al., Int. J. Mod. Phys. A20 (2005) 6932.

[10] V. Balkanov, et al., Nucl. Phys. (Proc. Suppl.) B91 (2001) 438.
[11] V. Agrawal, T. Gaisser, P. Lipari, T. Stanev, Phys. Rev. D53 (1996) 1314.

[12] K. Antipin, et al., in: Proceedings of the First Workshop on Exotic Physics with Neutrino Telescopes, Uppsala, Sweden, 2006, p. 34.

[13] V. Aynutdinov, et al., Nucl. Instr. and Meth. A588 (2008) 99.

[14] M. Boliev, et al., Nucl. Phys. (Proc. Suppl.) 48 (1996) 83; O. Suvorova, arXiv.org: hep-ph/9911415, 1999.

[15] M. Ambrosio, et al., Phys. Rev. D60 (1999) 082002.

[16] S. Desai, et al., Phys. Rev. D70 (2004) 083523; S. Desai, et al., Phys. Rev. D70 (2004) 109901 Erratum.

[17] J. Ahrens, et al., arXiv.org: astro-ph/0509330, 2005.

[18] V. Aynutdinov, et al., Astropart. Phys. 29 (2008) 366.

[19] S. Orito, et al., Phys. Rev. Lett. 66 (1991) 1951.

[20] M. Ambrosio, et al., Astropart. Phys. 19 (2003) 313.

[21] P. Niessen, C. Spiering, et al., in: Proceedings of the 27th ICRC, Hamburg, vol. 4, 2001, p. 1496.

[22] H. Wissing, et al., in: Proceedings of the 30th ICRC, Merida2007.

[23] M. Ackermann, et al., Astropart. Phys. 22 (2005) 127; M. Ackermann, et al., Astropart. Phys. 22 (2005) 339.

[24] M. Ackermann, et al., Astropart. Phys. 22 (2005) 339.

[25] M. Ackermann, et al., Phys. Rev. D76 (2007) 042008.

[26] F. Stecker, M. Salamon, Space Sci. Rev. 75 (1996) 341.

[27] F. Stecker, Phys. Rev. D72 (2005) 107301.

[28] A. Szabo, R. Protheroe, in: Proceedings of the High Energy Neutrino Astrophysics, Honolulu, HI1992.

[29] R. Protheroe, arXiv.org:astro-ph/9612213.

[30] K. Mannheim, Astropart. Phys. 3 (1995) 295.

[31] K. Mannheim, R. Protheroe, J. Rachen, Phys. Rev. D63 (2001) 023003.

[32] D. Semikoz, G. Sigl, arXiv.org:hep-ph/0309328.

[33] V. Aynutdinov, et al., in: Proceedings of the 29th ICRC, Pune, India, 2005 vol. 5, p. 231; arXiv.org: astro-ph /0507715.

[34] M. Ambrosio, et al., Nucl. Phys. (Proc. Suppl.) B110 (2002) 519.

[35] V. Berezinsky, et al., in: Astrophysics of Cosmic Rays, Elsevier, North-Holland, 1990.

[36] V. Berezinsky, arXiv.org: astro-ph/0505220, 2005.

[37] E. Waxman, J. Bahcall, Phys. Rev. D59 (1999) 023002.

[38] L. Nellen, K. Mannheim, P. Biermann, Phys. Rev. D47 (1993) 5270.

[39] L. Volkova, Yad. Fiz. 31 (1980) 1510.

[40] L. Volkova, G. Zatsepin, Phys. Lett. B462 (1999) 211.

[41] E. Bugaev, V. Naumov, S. Sinegovsky, E. Zaslavskaya, Nuovo Cim. C12 (1989) 41.

[42] V. Aynutdinov, et al., Izvestia RAN/Ser. Fiz. 3 (69) (2005) 406.

[43] J. Hörandel, Int. J. Mod. Phys. A20 (2005) 6753.

[44] V. Aynutdinov, et al., Int. J. Mod. Phys. A21S1 (2006) 117; V. Aynutdinov, et al., Acoust. Phys. 52 (5) (2006) 495.

[45] V. Aynutdinov, et al., in: Proceedings of the 30th ICRC, Merida, Mexico, 2007 vol. 5, p. 1561; arXiv.org: astro-ph/0710.3113.

[46] C.S. Clay, H. Medwin, Acoustical Oceanography, Wiley, New York, 1977.

[47] V. Aynutdinov, et al., in: Proceedings of the International ARENA2008 Workshop, Rome, 2008; Nucl. Instr. and Meth. A, 2009, in press.

[48] C.T. Chen, F.J. Millero, Limnol. Oceanogr. 31 (1986) 657.

[49] R.F. Weiss, E.C. Carmac, V.M. Koropalov, Nature 349 (1991) 665.

[50] V. Balkanov, et al., Appl. Opt. 38 (33) (1999) 6818.

[51] V. Balkanov, et al., Prog. Part. Nucl. Phys. 40 (1998) 391 astro-ph/9801044.

[52] I. Belolaptikov, et al., in: Proceedings of the 3rd Nestor International Workshop, Pylos, Greece, 1993; DESY-94-049.

[53] K. Kebkal, et al., 3D acoustic imaging applied to the Baikal neutrino telescope, these proceedings.

[54] M. Schmid, N.M. Budnev, N.G. Granin, et al., Geophys. Res. Lett. 35 (2008) L09605 10.1029/2008GL033223.

[55] V. Aynutdinov, et al., Nucl. Instr. and Meth. A 598 (2009) 282. 